\title{
The Israeli study of Pfizer BNT162b2 vaccine in pregnancy: considering maternal and neonatal benefits
}

\author{
Irina Burd, ${ }^{1}$ Tomoshige Kino, ${ }^{2}$ and James Segars ${ }^{3}$ \\ IIntegrated Research Center for Fetal Medicine, Department of Gynecology and Obstetrics, Johns Hopkins University School of Medicine, Baltimore, Maryland, USA. ${ }^{2}$ Laboratory of Molecular and Genomic \\ Endocrinology, Sidra Medicine, Doha, Qatar. ${ }^{3}$ Division of Reproductive Sciences, Department of Gynecology and Obstetrics, Johns Hopkins University School of Medicine, Baltimore, Maryland, USA.
}

Pregnant patients with COVID-19 are more likely to require intensive care and die compared with noninfected pregnant women. While the consequences of COVID-19 disease in pregnancy prompted many health care organizations to support vaccination in pregnancy, vaccine effects for mother and infant remained unclear. In this issue of the $J C l$, Beharier and Mayo et al. explored maternal and neonatal responses to the Pfizer BNT162b2 SARS-CoV-2 mRNA vaccine. The authors examined blood samples from women and cord blood of neonates following childbirth. Samples were stratified into three groups: vaccine recipients, unvaccinated participants with past positive SARS-CoV-2 test, and unvaccinated participants without prior infection. Vaccinated mothers and mothers with previous infection generated and transferred protective IgC antibodies across the placenta. This study provides evidence to support the safety and efficacy of COVID-19 vaccination in pregnancy with protection to the neonate against infection, outlining clear vaccine benefits for both maternal and child health.
Pregnancy shifts immune and endocrine function

Pregnancy is a state of altered immunity and endocrine function to permit the successful gestation and delivery of a semiallogeneic fetus. This shift in the immune and endocrine states during pregnancy promotes a vulnerability to severe complications caused by infectious agents, including SARS-CoV-2 infection. Specifically, pregnant patients are three-fold more likely to be admitted to an intensive care unit, to receive ventilator support or extracorporeal membrane oxygenation, and die compared with noninfected pregnant women $(1,2)$. Additionally, obstetrical complications, in particular preterm birth $(2,3)$, are also more likely to occur if the mother is infected with SARS-CoV-2, thus increasing the possibility of long-term health problems for both the mother and the neonate.
Because of the novelty and associated uncertain efficacy of the mRNA COVID-19 vaccines, pregnancy was an exclusion criterion from clinical trials of these vaccines. Given the grave consequences of COVID-19 disease in pregnancy, many health care societies and governmental organizations began to advocate for administration of the vaccine in pregnancy for the health of pregnant patients and their unborn children. In the United States, the American Society for Reproductive Medicine (ASRM), the American College of Obstetricians and Gynecologists (ACOG), and the Society for MaternalFetal Medicine (SMFM) supported vaccination, in accordance with the Advisory Committee for Immunization Practices of the US Centers for Disease Prevention and Control (CDC). Weighing the known risks and benefits and considering possi-

\section{Related Article: https://doi.org/10.1172/JCl150319}

Conflict of interest: The authors have declared that no conflict of interest exists.

ble unknown risks based on a deep understanding of the physiologic immune and endocrine shifts of pregnancy, the various committees and their task force members awaited more data, understanding that the emergency use authorization (EUA) issued by the FDA permitted vaccination of pregnant and breastfeeding individuals erstwhile requiring that the company conduct postauthorization observational studies in pregnancy (4). Current evidence indicates that COVID-19 vaccines do not precipitate infertility in women or men, and while administered in pregnancy, the vaccinated mRNA fragment does not cross the placenta, being consumed by muscle cells at the site of injection and degraded after the encoded protein is synthesized (5-7). However, though reassuring, data pertaining to pregnancy and neonatal transfer are still limited.

\section{Maternal and neonatal responses}

In this issue of the JCI, Beharier and Mayo et al. (8) provide much needed information regarding maternal and neonatal responses to the Pfizer BNT162b2 SARS-CoV-2 mRNA vaccine in pregnancy, capitalizing on the unprecedented COVID-19 vaccination campaign in Israel, which included pregnant women. The investigators examined a cohort of 1094 participants from eight hospitals across Israel. Blood samples from women and cord blood of neonates following childbirth were collected between April 2020 and March 2021. Samples were stratified into three groups: 105 vaccine recipients (samples collected between January to March 2021), 94 unvaccinated participants with past positive results in SARS-CoV-2 RT-PCR test, and 895 unvaccinated participants without prior documentation for infection. A positive PCR test was considered diagnostic of acute infection. The authors used multiplexed serological IgG and IgM responses to the S1 domain, S2 domain, receptor 


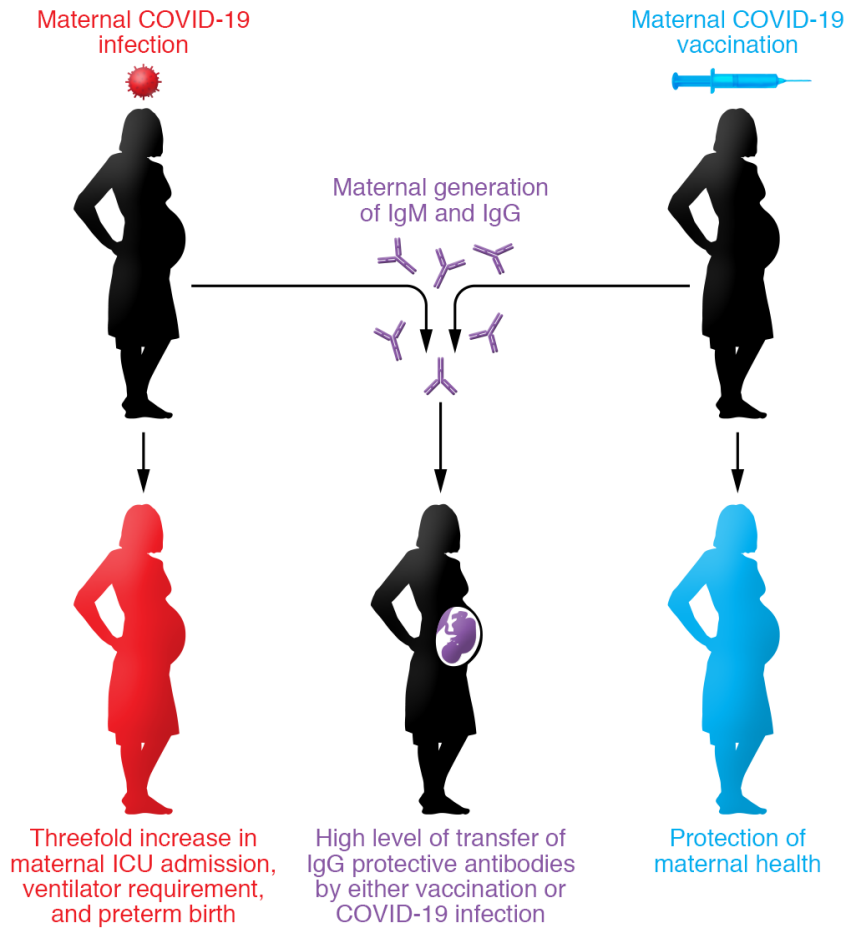

binding domain (RBD), and nucleocapsid $(\mathrm{N})$ antigens in maternal and neonatal sera to identify the antiviral immunity induced by the vaccination and to determine the participants with a previous infection. A response to $\mathrm{N}$ (anti- $\mathrm{N}$ antibody) was present in SARS-CoV-2-infected subjects, but not in the BNT162b2 mRNA vaccinated. Conversely, a response to RBD (anti-RBD antibody) was present in both infected and vaccinated subjects.

\section{Successful transfer from mother to fetus}

There were several notable findings that expanded understanding of COVID-19 vaccination in pregnancy. The data showed a robust increase in maternal IgG humoral response against $\mathrm{S} 1, \mathrm{~S} 2$, and $\mathrm{RBD}$ in noninfected patients upon vaccination. These immunoglobulins were readily transferred to the fetus across the placenta, leading to a substantial anti-SARS-CoV-2 antibody titer in the neonatal bloodstream within 14 days of the first vaccine dose. In addition, in women recovering from SARSCoV-2 infection contracted in the second trimester (13-26 weeks of gestation), both maternal and cord blood anti-COVID-19 antibodies remained elevated at delivery. Overall, there was a high efficacy of the vaccination to generate protective antibodies (S1, S2, and RBD IgGs) in mothers and subsequent successful transfer with fetuses compared with natural infection (Figure 1 and ref. 8). IgG is the only antibody class that is actively transferred from the mother to the fetus across the placenta by an active transport, involving neonatal Fc receptor ( $\mathrm{FcRn})$, providing protection against SARS-CoV-2 to the newborn during the first months of life. In the 86 vaccinated deliveries, there was no evidence of fetal IgM response to any of the vaccine-induced antigens, indicating no evidence for direct exposure of the fetus to vaccine-derived antigens (8). We commend Beharier, Mayo, and colleagues for addressing the important question of COVID-19 vaccination and antibody transfer to the fetus.

\section{Conclusions and considerations}

As noted by Beharier, Mayo, and their co-authors, some patients were excluded, possibly including cases of acute infection in pregnancy. Also, the race and ethnicity were limited based on the population, and differences might be observed in other ethnicities (8). Future studies are urgently needed to address this point since race and ethnicity are known to increase the risk of adverse pregnancy outcomes with SARSCoV-2 infection (9).

One point not directly addressed by the authors is that FcRn receptor expres-
Figure 1. Pfizer BNT162b2 vaccine in pregnancy provides maternal and neonatal benefits. Pregnant women with COVID-19 are three times more likely to suffer complications that may include ICU admission, ventilator requirements, or preterm birth. Beharier and Mayo et al. showed that mothers with previous SARS-CoV-2 infection and vaccinated mothers generate and transfer protective IgG antibodies across the placenta to the neonate (8). sion in the placenta is not constant during pregnancy (8). The expression of the FcRn receptor depends on gestational age and is highest at the end of pregnancy (10-12). In placenta, these receptors are mainly located on the syncytiotrophoblasts. At early stages of pregnancy, the continuous cytotrophoblast layer between syncytiotrophoblasts and stromal cells prevents penetration of the villi by IgG. After the fourth month of pregnancy, the cytotrophoblast layer becomes discontinuous, slowly disappearing through the remaining gestation, and exposes the syncytiotrophoblast cells to maternal blood vessels so that the active transport of IgG may begin. This fact suggests that vaccination in very early pregnancy might not transfer maternally generated protective antibodies to the fetus with an efficiency similar to vaccination later in pregnancy. In the view of the authors of this Commentary, however, this point does not detract from endorsement of vaccination at any time during gestation because vaccination in pregnancy confers separate benefits to the mother and the fetus. The health of both the fetus and mother must be considered. Because of the considerable burden of disease throughout pregnancy on the mother, vaccinations at any time of gestation should be strongly considered for the benefit of maternal health. 
Address correspondence to: James Segars, Ross Building, Room 624, 720 Rutland Avenue, Baltimore, Maryland 21205, USA. Phone: 410.614.2000; Email: jsegars2@ jhmi.edu.

1. Zambrano LD, et al. Update: characteristics of symptomatic women of reproductive age with laboratory-confirmed SARS-CoV-2 Infection by pregnancy status - United States, January 22October 3, 2020. MMWR Morb Mortal Wkly Rep. 2020;69(44):1641-1647.

2. Allotey J, et al. Clinical manifestations, risk factors, and maternal and perinatal outcomes of coronavirus disease 2019 in pregnancy: living systematic review and meta-analysis. BMJ. 2020;370:3320.

3. Woodworth KR, et al. Birth and infant outcomes following laboratory-confirmed
SARS-CoV-2 infection in pregnancy SET-NET, 16 jurisdictions, March 29-October 14, 2020. MMWR Morb Mortal Wkly Rep. 2020;69(44):1635-1640.

4. Food and Drug Administration. Pfizer-BioNTech COVID-19 vaccine EUA letter of authorization reissued 05-10-2021. https://www.fda.gov/ media/144412/download. Updated May 10, 2021. Accessed May 27, 2021.

5. Centers for Disease Control and Prevention. Understanding COVID-19 vaccines. https:// www.cdc.gov/coronavirus/2019-ncov/vaccines/ different-vaccines/mrna.html. Updated May 4, 2021. Accessed May 27, 2021.

6. Hajissa K, Mussa A. Positive aspects of the mRNA platform for SARS-CoV-2 vaccines [published online April 8, 2021. Hum Vaccin Immunother. https://doi.org/10.1080/ 21645515.2021.1900713.

7. Anderson EJ, et al. mRNA1273 study group.
Safety and immunogenicity of SARS-CoV-2 mRNA-1273 vaccine in older adults. $N$ Engl J Med. 2020;383(25):2427-2438.

8. Beharier O, et al. Efficient maternal to neonatal transfer of antibodies against SARS-CoV-2 and BNT162b2 mRNA COVID-19 vaccine. JClin Invest. 2021;131(13):e150319.

9. Gur RE, et al. The disproportionate burden of the COVID-19 Pandemic among pregnant black women. Psychiatry Res. 2020;293:113475.

10. Palmeira $P$, et al. IgG placental transfer in healthy and pathological pregnancies. Clin Dev Immunol. 2012;2012:1-13.

11. Lozano NA, et al. Expression of FcRn receptor in placental tissue and its relationship with IgG levels in term and pre-term newborns. Am J Reprod Immunol. 2018; 80(3):e12972.

12. Rath $\mathrm{T}$, et al. Regulation of immune responses by the neonatal Fc receptor and its therapeutic implications. Front Immunol. 2015;5(664):1-8. 